\title{
The Subaru coronagraphic extreme AO (SCExAO) system: wavefront control and detection of exoplanets with coherent light modulation in the focal plane
}

Guyon, Olivier, Martinache, Frantz, Garrel, Vincent, Vogt, Frederic, Yokochi, Kaito, et al.

Olivier Guyon, Frantz Martinache, Vincent Garrel, Frederic Vogt, Kaito Yokochi, Takashi Yoshikawa, "The Subaru coronagraphic extreme AO (SCExAO) system: wavefront control and detection of exoplanets with coherent light modulation in the focal plane," Proc. SPIE 7736, Adaptive Optics Systems II, 773624 (14 July 2010); doi: 10.1117/12.857878

Event: SPIE Astronomical Telescopes + Instrumentation, 2010, San Diego, California, United States 


\title{
The Subaru Coronagraphic Extreme AO (SCExAO) System: Wavefront Control and Detection of Exoplanets with Coherent Light Modulation in the Focal Plane
}

\author{
Olivier Guyon ${ }^{\mathrm{a}}$, Frantz Martinache ${ }^{\mathrm{a}}$, Vincent Garrel ${ }^{\mathrm{a}}$, Frederic Vogt ${ }^{\mathrm{a}}$, Kaito Yokochi ${ }^{\mathrm{a}}$, Takashi \\ Yoshikawa $^{a}$ \\ ${ }^{\text {a}}$ Subaru Telescope, 650 N. A'ohoku Place, Hilo, HI 96720
}

\begin{abstract}
The Subaru Coronagraphic Extreme-AO (SCExAO) system is designed for high contrast coronagraphic imaging at small angular separations, and is scheduled to see first light on the Subaru Telescope in early 2011. The wavefront control architecture for SCExAO is optimized for scattered light control and calibration at small angular separations, and is described in this paper. Key subsystems for the SCExAO wavefront control architecture have been successfully demonstrated, and we report results from these tests and discuss their role in the SCExAO system. Among these subsystems, a technique which can calibrate and remove static and slow speckles which traditionally limit high contrast detections is discussed. A visible light lab prototype system at Subaru Telescope recently demonstrated speckle halo reduction to $2 \mathrm{e}-7$ contrast within $2 \lambda / \mathrm{D}$, and removal of static coherent speckles to $3 \mathrm{e}-9$ contrast.
\end{abstract}

Keywords: Exoplanets, Coronagraphy, Adaptive Optics

\section{SUBARU CORONAGRAPHIC EXTREME-AO SYSTEM OVERVIEW}

The Subaru Coronagraphic Extreme-AO (SCExAO) system is a platform dedicated to high contrast imaging at small angular separation from stars. Its main goals are to directly image and characterize extrasolar planets and disks. It is scheduled to be installed on the 8-m Subaru Telescope in early 2011 for first light ${ }^{1}$. The SCExAO platform takes advantage of the existing AO188 facility adaptive optics and the HiCIAO near-camera specifically designed for high contrast imaging. The SCExAO bench takes light from AO188, performs further wavefront correction/calibration and coronagraphic suppression of starlight, and uses the HiCIAO near-IR camera as a science detector. A separate visible light channel is used for both visible wavefront sensing and visible light imaging ${ }^{2}$.

SCExAO is a highly flexible platform, allowing fast upgrade and considerably reducing the time required to implement new techniques. This is a key advantage for high contrast imaging, where new techniques are constantly under development. SCExAO is optimized for delivering high contrast in the 1 to $10 \lambda / \mathrm{D}$ separation range in H-band, and uses coronagraphic and wavefront control techniques well suited for small angular separations:

- SCExAO's coronagraph, based on the PIAA technique ${ }^{3-5}$, allows high contrast imaging at $1 \lambda / \mathrm{D}$ without loss in efficiency or angular resolution

- SCEcAO's wavefront control architecture, discussed in this paper, is optimized for precise control of low order aberrations, which is key to achieving high contrast at small angular resolution

\section{WAVEFRONT CONTROL ARCHITECTURE OVERVIEW}

The SCExAO wavefront control architecture is shown in figure 1. It is highly integrated with the coronagraph architecture. The SCExAO system relies on a total of 4 wavefront sensors for wavefront control: one within the AO188 system $^{6}$ and 3 within SCExAO/HiCIAO. Out of the 3 WFSs within SCExAO/HiCIAO, one is located ahead of the coronagraph (ExAO visible WFS), one is located within the coronagraph (CLOWFS) and one is located downstream of the coronagraph (focal plane WFS). Thanks to this combination of WFSs, speckles in the final science image are both minimized in intensity and well calibrated. The list of WFSs and their roles are briefly listed in Table 1, and further described in sections of this paper.

Adaptive Optics Systems II, edited by Brent L. Ellerbroek, Michael Hart, Norbert Hubin, Peter L. Wizinowich, Proc. of SPIE Vol. 7736, 773624 - (c) 2010 SPIE · CCC code: 0277-786X/10/\$18 · doi: 10.1117/12.857878 
A key architecture choice for the SCExAO system is to separate AO188 from the rest of the system: the AO188 "woofer" is fully decoupled from the SCExAO "tweeter". This choice facilitates sytem integration and testing, as changes to the facility AO188 system are delicate due to the fact that AO188 is a general purpose AO system in high demand.

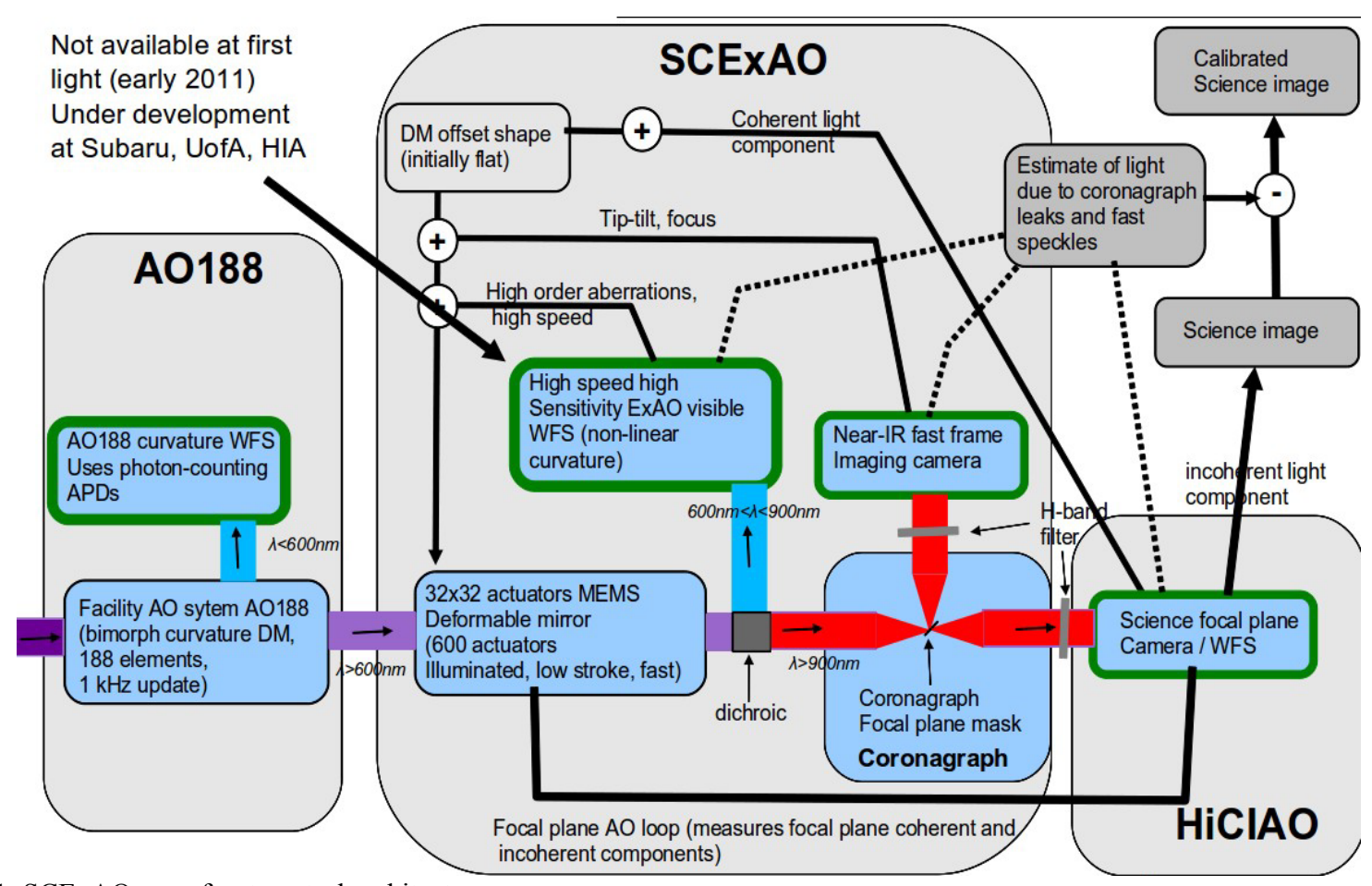

Figure 1: SCExAO wavefront control architecture.

Table 1: List of WFSs in the SCExAO system wavefront control architecture.

\begin{tabular}{|c|c|c|c|c|}
\hline Wavefront sensor & $\begin{array}{l}\text { Light used } \\
\text { [sampling speed] }\end{array}$ & Signal fed to ... & role & notes \\
\hline AO188 curvature WFS & $\begin{array}{l}\lambda<600 \mathrm{~nm} \\
{[1 \mathrm{kHz}]}\end{array}$ & AO188 bimorph DM & $\begin{array}{l}\text { System "woofer": } \\
\text { reduces wavefront errors } \\
\text { to } \sim 200 \mathrm{~nm}\end{array}$ & $\begin{array}{l}\text { Part of AO188 system, } \\
\text { functions independently } \\
\text { of SCExAO }\end{array}$ \\
\hline SCExAO visible WFS & $\begin{array}{l}600 \mathrm{~nm}<\lambda<900 \mathrm{~nm} \\
{[1-5 \mathrm{kHz}]}\end{array}$ & $\begin{array}{l}\text { SCExAO MEMS (high } \\
\text { speed component) }\end{array}$ & $\begin{array}{l}\text { System "tweeter": high } \\
\text { order wavefront } \\
\text { correction optimized for } \\
\text { ExAO, with excellent } \\
\text { sensitivity to low-order } \\
\text { modes }\end{array}$ & $\begin{array}{l}\text { Under development } \\
\text { See section } 3\end{array}$ \\
\hline \multirow[t]{2}{*}{ LOWFS } & \multirow{2}{*}{$\begin{array}{l}\text { Near-IR } \\
\text { light within } 1 \lambda / \mathrm{D} \\
{[1 \mathrm{kHz}]}\end{array}$} & $\begin{array}{l}\text { SCExAO MEMS (low } \\
\text { orders) }\end{array}$ & \multirow{2}{*}{$\begin{array}{l}\text { Measure and control low } \\
\text { order aberrations in } \\
\text { coronagraph at science } \\
\text { wavelength }\end{array}$} & \multirow[t]{2}{*}{ See section 4} \\
\hline & & PSF calibration & & \\
\hline \multirow{2}{*}{$\begin{array}{l}\text { Focal plane AO using } \\
\text { science Camera } \\
\text { (HiCIAO or internal } \\
\text { SCEXAO science } \\
\text { camera) }\end{array}$} & \multirow[t]{2}{*}{$\begin{array}{l}\text { Near-IR } \\
\text { light outside } 1 \lambda / \mathrm{D} \\
{[10 \mathrm{~Hz}-500 \mathrm{~Hz}]}\end{array}$} & $\begin{array}{l}\text { SCExAO MEMS (high } \\
\text { orders, low speed } \\
\text { component) }\end{array}$ & \multirow{2}{*}{$\begin{array}{l}\text { Calibrate and remove } \\
\text { non-common path errors } \\
\text { and slow speckles at } \\
\text { science wavelength. Test } \\
\text { coherence of residual } \\
\text { speckles }\end{array}$} & \multirow[t]{2}{*}{ See section 5} \\
\hline & & PSF calibration & & \\
\hline
\end{tabular}




\section{HIGH SPEED, HIGH SENSITIVITY VISIBLE WAVEFONT SENSOR}

The beam from the AO188 contains approximately $200 \mathrm{~nm}$ of residual wavefront error. This level of residual wavefront is due to the limited number of elements in the AO188 system, and to the fact that AO188 is a general-purpose facility AO system which is not optimized for Extreme-AO (high level of correction on bright natural guide stars).

High contrast imaging requires exquisite control of low order aberrations. Conventional wavefront sensors, such as the Shack-Hartmann wavefront sensor (SHWFS) and the conventional curvature wavefront sensor (CWFS), are very innefficient for measuring low order aberrations. This is fundamentally due to the fact that they are seeing-limited and do not take advantage of coherence across the pupil ${ }^{7}$. For example, a Shack-Hartmann wavefront sensor measuring tip-tilt with a total of $\mathrm{N}$ photon in the pupil will essentially measure the location of a seeing-sized spot with $\mathrm{N}$ photon, and therefore estimate tip tilt with $\sim\left(\lambda / \mathrm{r}_{0}\right) / \mathrm{sqrt}(\mathrm{N})$ accuracy. A WFS operating at the diffraction limit of the telescope, instead, will estimate tip-tilt $\sim(\lambda / \mathrm{D}) / \mathrm{sqrt}(\mathrm{N})$ accuracy. The difference in sensitivity, when expessed in equivalent number of photon, is $\left(\mathrm{D} / \mathrm{r}_{0}\right)^{2}$ : on a $8-\mathrm{m}$ telescope with $\mathrm{r}_{0}=15 \mathrm{~cm}$, a conventional seeing-limited WFS such as the SHWFS is approximately 3000 times less efficient than a WFS operating at the diffraction limit of the telescope for tip-tilt sensing. This difference in efficiency is especially large for low-order aberrations, which are the most important for high contrast coronagraphic imaging.

There are a few WFS concepts which operate at the diffraction limit of the telescope: pyramid wavefront sensor (when non-modulated), non-linear curvature wavefront sensor, Zerkine phase mask wavefront sensor. We have adopted the non-linear curvature WFS (nlCWFS) for SCExAO because it is the only WFS which can maintain high diffractionlimited efficiency with moderate levels of wavefront aberrations. Unlike the pyramid wavefront sensor, it does not require high Strehl ratio at the sensing wavelength to offer diffraction-limited sensitivity. The nlCWFS principle is described in separate publications ${ }^{8,9}$. As shown in figure 2 , four images are acquired in planes near the pupil plane (panels (a) to (d)). These images show diffraction-limited speckles which encode wavefront aberrations with high sensitivity. The relationship between these speckles and the wavefront phase is non-linear over a large range of input phase, but is locally (within 1 radian) linear around any wavefront phase map. The reconstruction algorithm is therefore semi-linear, with initial solving of the wavefront map requiring a non-linear iterative solver, while closed-loop ExAO wavefronts can be solved with a faster algorithm utilizing local linearity. Figure 2 shows that with a limited available number of photons, the result of the reconstruction is highly accurate.
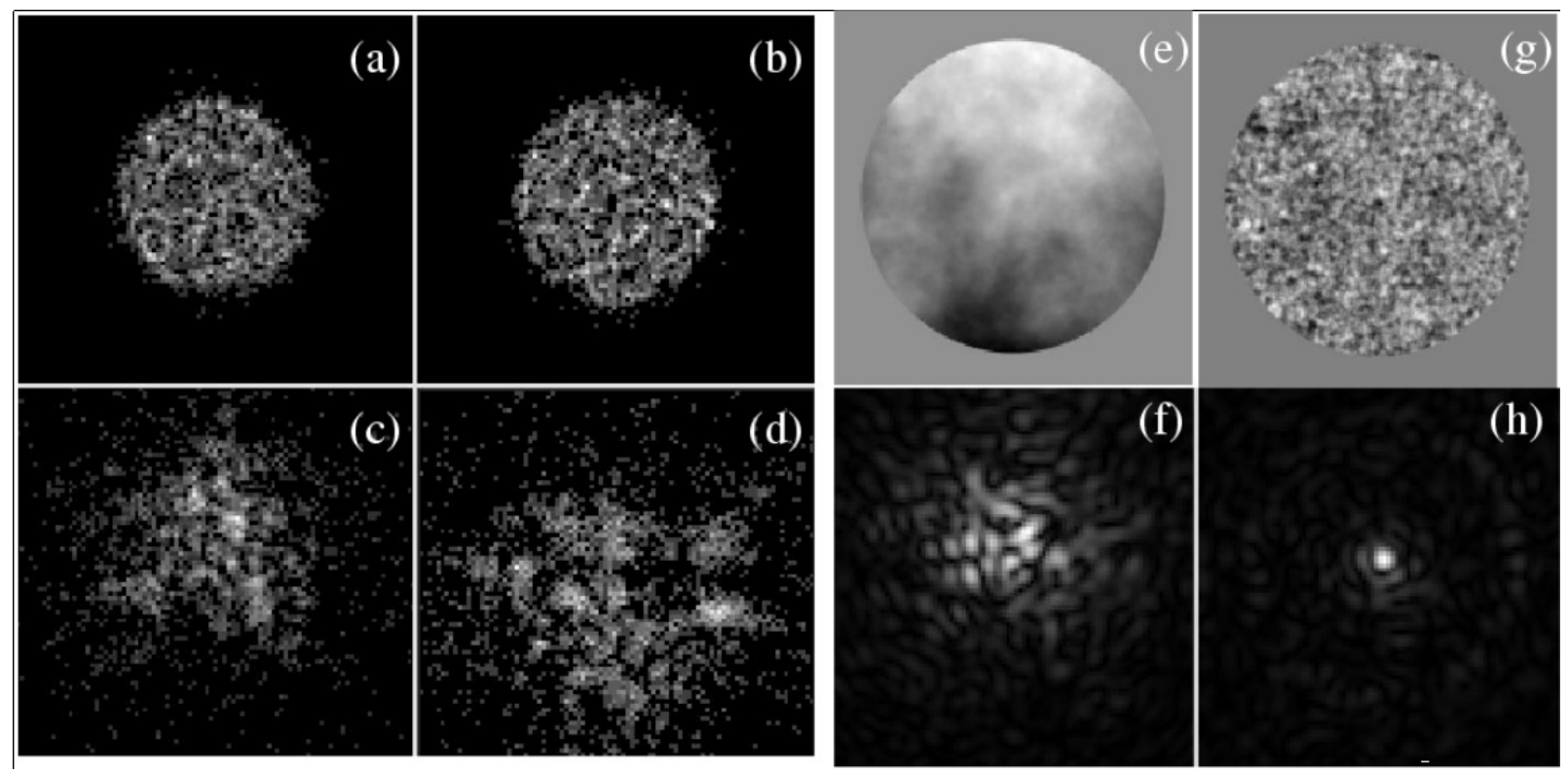

Figure 2: nlCWFS: data acquisition and wavefront reconstruction. The nlCWFS data consists of four defocused pupil images ((a) to (d)). Photon noise is visible in the data, which contains only 20000 photons (split between the four images). The input wavefront with $604 \mathrm{~nm}$ RMS phase error (e) is well estimated, and the residual wavefront error (g) is $34.4 \mathrm{~nm}$. The corresponding PSFs before (f) and after (h) correction are shown at $=0.65 \mu \mathrm{m}$. 
Closed-loop simulations with the nlCWFS demonstrate the benefit of using nlCWFS over a conventional SHWFS. As shown in figure 3 , the nlCWFS allows aliasing-free high sensitivity wavefront reconstruction. The performance advantage is especially large at small angular separation, where the closed-loop PSF shows well defined Airy rings which can be efficiently removed by the coronagraph. In comparison, with a SHWFS, the inner part of the PSF lack contrast due to residual low-order aberrations.

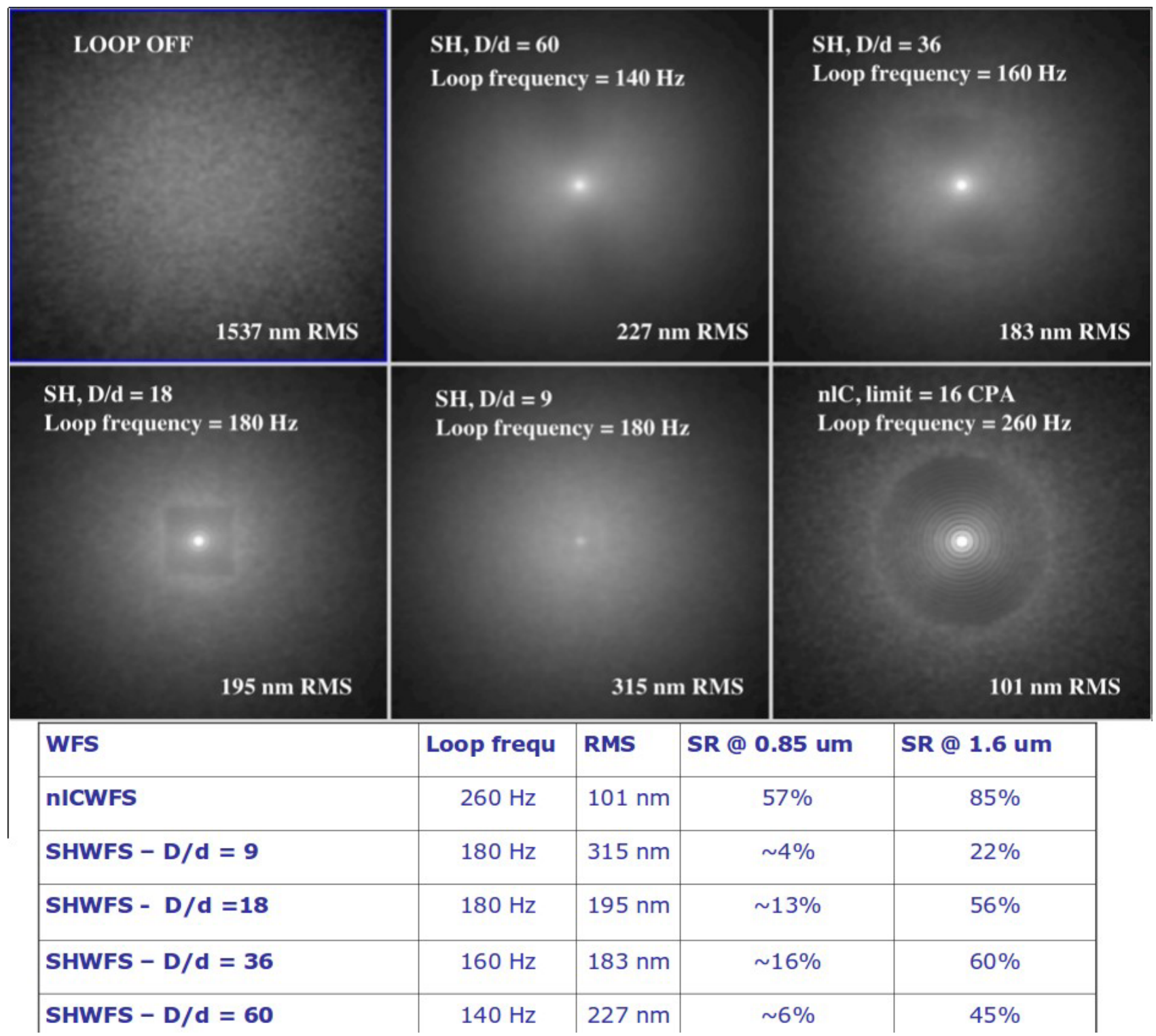

Figure 3: Comparison between nICWFS and SHWFS. This closed loop simulation shows PSFs for the observation of a $\mathrm{m}_{\mathrm{V}}=13$ star with an $8-\mathrm{m}$ telescope. The nlCWFS is compared to several SHWFSs with varying subpupil sizes d. The nlWFS offers better sensitivity, and can therefore run faster with the same total number of photon. The nlWFS PSF shows superior control of low order aberrations and more well-defined Airy rings.

\section{CORONAGRAPHIC LOW ORDER WAVEFRONT SENSOR (CLOWFS)}

Control of low-order aberrations is essential for high contrast imaging at small angular separations. Small levels of residual tip-tilt or focus errors create coronagraph leaks around the coronagraph focal plane mask, which is the region of most scientific interest. It is therefore essential to both: 
(1) minimize low order aberrations to reduce starlight in the region of scientific interest

(2) have a good knowledge of the residual low order aberrations during the science observation to avoid confusion between real sources (planets, disk) and coronagraphic leaks induced by wavefront errors

The second point is often overlooked but is extremely important, as a small pointing excursion produces an image which looks almost identical to a planet at near the inner-working angle of the coronagraph.

To meet this requirement, SCExAO uses a dedicated coronagraphic low-order wavefront sensor (CLOWFS). CLOWS operates at the same wavelength as the science camera: this is essential to avoid chromatic differences between the measured aberrations and the aberrations-induced coronagraphic leaks seen by the science camera. The CLOWFS principle, shown in figure 4 , is detailed in previous publications ${ }^{10,11}$.

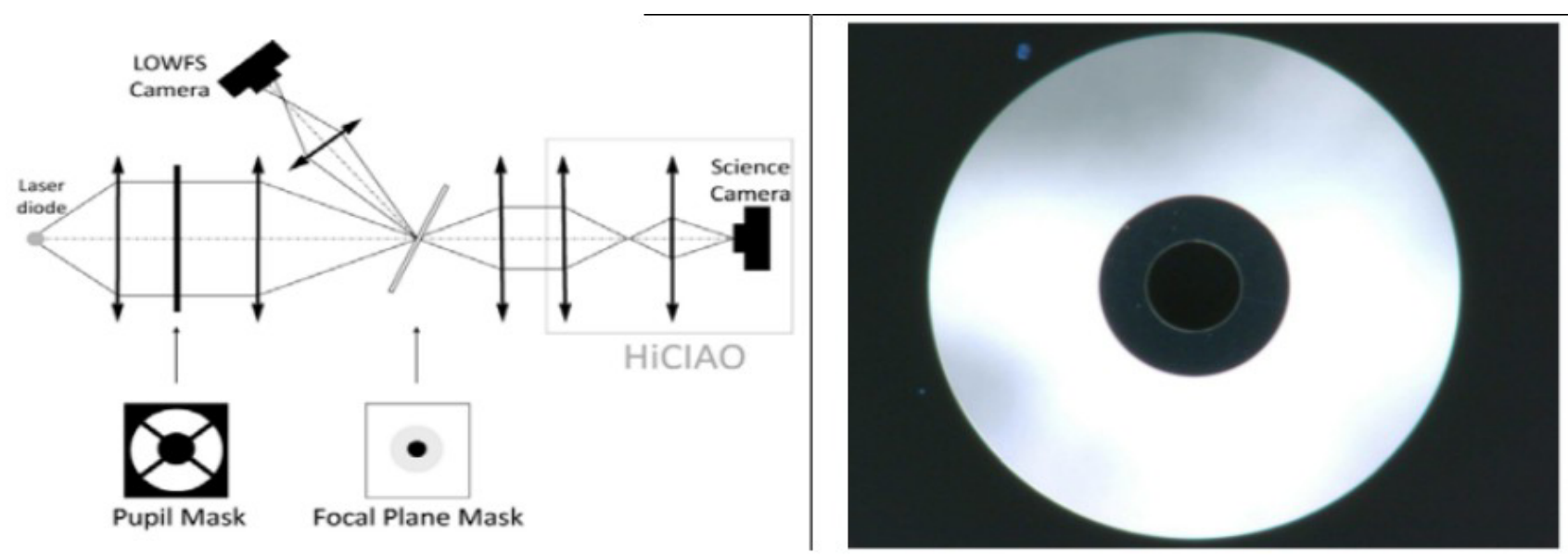

Figure 4: CLOWFS principle. The CLOWFS uses light reflected by the coronagraph focal plane mask to measure low order aberrations. The focal plane mask contains a dark central zone surrounded by a reflective annulus: this design allows high precision measurement of low order aberrations free of non-common path errors.

Closed loop control of low order aberrations: laboratory demonstration: The first goal of the CLOWFS is to drive the SCExAO DM and its tip-tilt platform to keep low-order aberrations small in the system (figure 5, top half). We have performed visible light demonstration of closed-loop control of low-order aberrations with a PIAA coronagraph. In this laboratory validation, 5 low-order modes were simultaneously controlled. A 1e-3 $\lambda / \mathrm{D}$ closed loop residual was obtained for tip and tilt. The results of this experiment are described in a previous publication ${ }^{10}$. This performance exceeds the requirement for SCExAO. The SCExAO CLOWFS system operates in near-IR with a fast camera reading a small 10x10 pixels window at approximately $1 \mathrm{kHz}$.

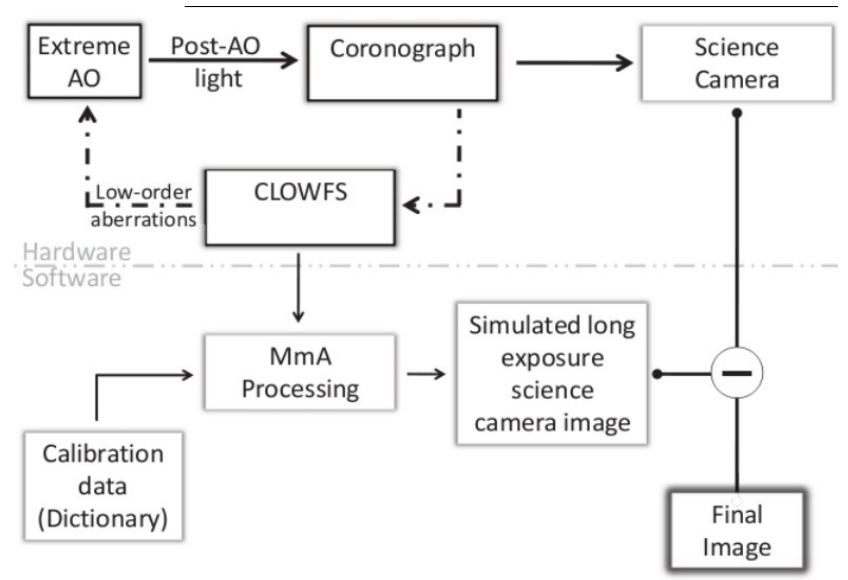

Figure 5: The CLOWFS is used in SCExAO to drive a closed-loop control of low order aberrations (top half of the figure) and to remove, in post-processing, the contribution of low-order aberrations to the final image (bottom half of the figure). 
Calibration of coronagraphic leaks with the CLOWFS: laboratory demonstration. The second goal of the CLOWFS is to measure residual low order aberrations for post-processing of the science images (figure 5, bottom half). With a good knowledge of the temporal evolution of low-order aberrations during the science exposure, it is possible to estimate the contribution of the low-order aberration induced coronagraphic leaks in the final science image. This contribution is then substracted from the science image. This post-processing loop was successfully validated on the SCExAO bench, and led to almost a 100x reduction in scattered light in the calibrated science image ${ }^{11}$.

\section{FOCAL PLANE COHERENT LIGHT MODULATION (FPCLM)}

Thanks to the AO188 system and the SCExAO visible WFS, the wavefront at the input of the coronagraph is wellcontrolled, and the CLOWFS loop keeps low order aberrations small. These subsystems are however not able to fully remove slow and static wavefront errors in the near IR mainly due to :

- wavefront chromaticity: the high speed ExAO visible WFS operates in the visible, while the science image is acquired in the near-IR. Chromaticity in the atmosphere and the optics in the system lead to small differences between the OPD in the visible and the OPD in the near-IR

- non-common path errors: the visible WFS does not correct for wavefront aberrations introduced after the visible/nearIR dichroic in the system, or for wavefront errors in the WFS optics

- aliasing: While the visible WFS suffers from little aliasing, there is some finite amount of coupling between the measurement of low order aberrations and high spatial frequency aberrations.

For these reasons, the near-IR science image is expected to contain static and slow near-IR speckles. While these speckles are well below the halo of fast atmospheric speckles, they would be visible in long exposures because they do not average well with exposure time, and they are especially difficult to distinguish from actual sources (planets). These static and quasi-static speckles are currently the limiting factor in high contrast imaging.

To address this issue, SCExAO uses focal plane coherent light modulation (FPCLM) of the speckles to identify and remove speckles in the focal plane ${ }^{12}$. This technique has been successfully used on several high contrast testbeds, and uses a DM ahead of the coronagraph (a 32x32 actuators MEMS for SCExAO) to create focal plane speckles at will. A single speckle is created by adding a sine wave pattern to the DM: the amplitude of the pattern is proportional to the square root of the speckle intensity, the frequency and orientation of the sine wave sets the speckle location in the image, and its phase encodes the phase of the speckle. An complex amplitude field can be added in the focal plane by linearly decomposing it in a sum of speckles and adding the corresponding sum of sine waves to the DM. The closed loop coherent light modulation based on this principle is described in figure 6, and works as follows:

- Step 1: A focal plane image is acquired $\left(\mathrm{I}_{0}{ }^{\mathrm{k}}\right)$. Speckles are identified from this image, but their phase is unknown.

- Step 2: From image \#1, a DM shape offset $\delta \mathrm{DM}_{1}{ }^{\mathrm{k}}$ is computed to create in the focal plane the same speckle intensity map as measured in the first image, but with a known phase. This DM shape offset is applied and a new image is acquired $\left(\mathrm{I}_{1}^{\mathrm{k}}\right)$

- Step 3: Another set of DM shape offset $\delta \mathrm{DM}_{2}{ }^{\mathrm{k}}$ is computed and applied to the DM to add to the focal plane the same speckle pattern as added in step 2, but with a quarter-wave offset in phase compared to the pattern added in step 2. The corresponding image is then acquired $\left(\mathrm{I}_{2}{ }^{\mathrm{k}}\right)$

- Step 3' (optional): More images $\mathrm{I}_{3}{ }^{\mathrm{k}} \ldots \mathrm{I}_{\mathrm{n}}{ }^{\mathrm{k}}$ can be acquired with different DM shape settings. This can allow a more robust estimation of the wavefront aberration and more diagnostics, at the expense of a slower loop speed.

- Step 4: With images $\mathrm{I}_{0}{ }^{\mathrm{k}}, \mathrm{I}_{1}{ }^{\mathrm{k}}, \mathrm{I}_{2}{ }^{\mathrm{k}}$ (and possibly more images), the speckle complex amplitude in image $\mathrm{I}_{0}{ }^{\mathrm{k}}$ is computed, and the DM shape offset necessary to cancel it is applied the DM. If more than 3 images are available, both the coherent and incoherent parts of the focal plane light can be computed: in this case, only the coherent part of the light is used to compute the new DM offset (the incoherent part of the light cannot be modified with a DM offset).

When this algorithm is running in a loop, a full iteration consists of these 4 (or more if optional images are acquired) steps, which are repeated. By post-processing of the focal plane images and the DM commands, it is possible to also calibrate the residual light in the focal plane, and estimate how much it changes between individual frames, and what is the fraction of the focal plane light which is coherent vs. incoherent. 


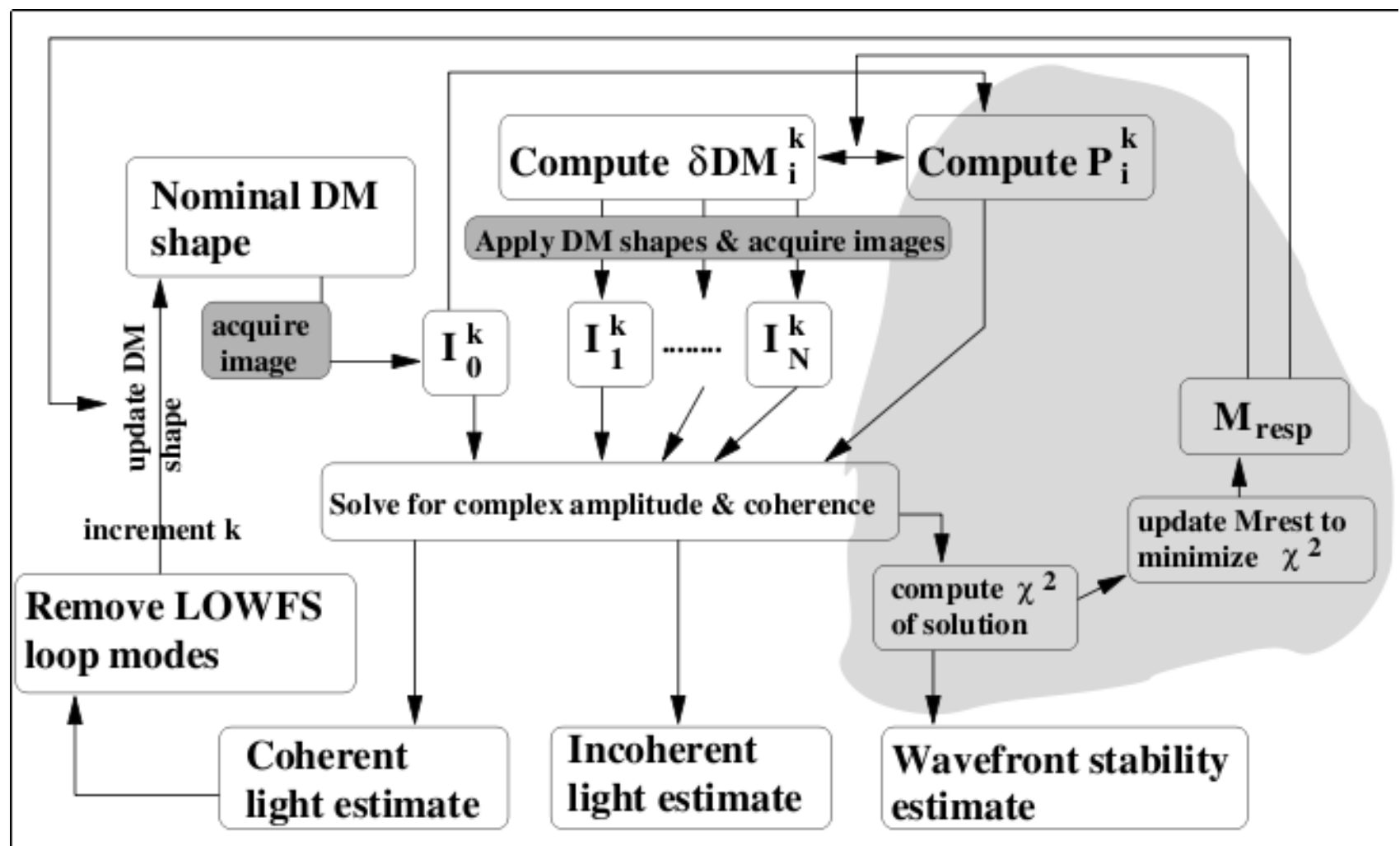

Figure 6: Focal plane coherent light modulation loop for SCExAO. By analysis of focal plane images acquired with different DM shapes, the system can estimate coherent and incoherent light in the focal plane as well as wavefront variations between individual frames.

The residual coherent light estimate wavefront stability estimate are used towards estimating the contribution of uncorrected wavefront aberrations to the science image, providing a further level of speckle calibration. The FPCLM algorithm also includes a self-tuning loop, where the data acquired is used to constrain a model of the coronagraph system which is used to convert DM commands into focal plane complex amplitudes (grey area on the right of figure 6).

\section{Laboratory validation}

The focal plane coherent light modulation loop described in figure 6 was implemented on the PIAA coronagraph prototype at the Subaru Telescope laboratory. The experiment was conducted in air within a temperature-stabilized air enclosure in visible light. The key goals of this of this experiment are :

- to validate that focal plane coherent modulation can run with a PIAA type coronagraph and remove static speckles

- to demonstrate that the control loop is able to separate coherent light and incoherent light

- to verify that the static and slow speckles can be reduced well below the dynamic speckle halo due to fast turbulence, and that the reliable estimation of static and slow speckles is still possible in the presence of much stronger dynamic aberration (this is the situation under which SCExAO is expected to operate)

Details of the experiment and its results can be found in a separate publication ${ }^{5}$. We summarize here the results obtained and discuss their validity to the SCExAO wavefront control architecture. Figure 7 shows that the 3 key requirements outlined above were validated. Of particular interest is the ability of the FPCLM technique to reduce slow and static speckles well below the dynamic speckle halo level. In the laboratory experiment, the dynamic coherent speckle halo was at $5 \mathrm{e}-8$ contrast level, and the measured averaged complex amplitude (residual static speckles) over 1300 loop iterations was at the $3.5 \mathrm{e}-9$ contrast level. 

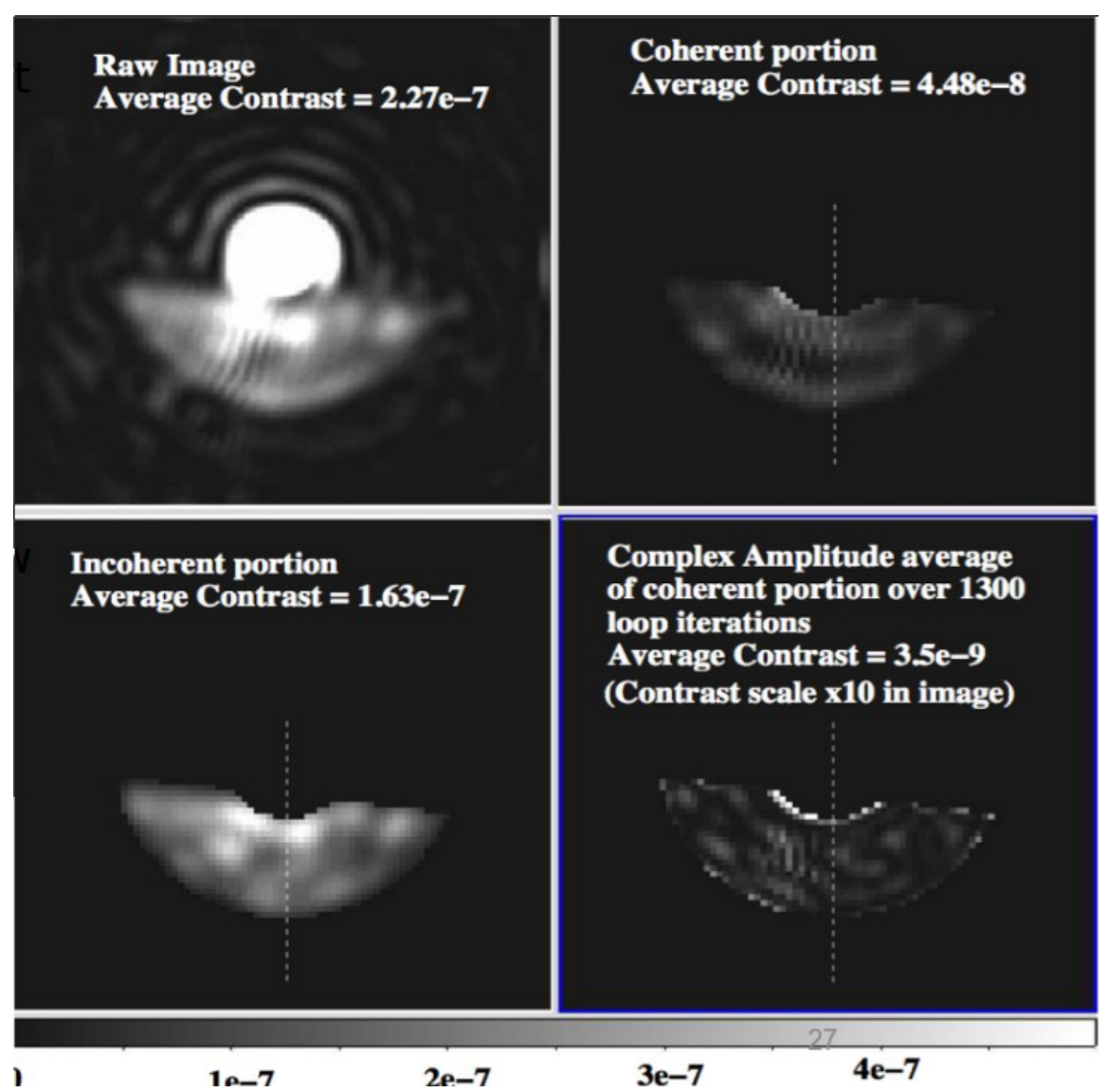

Figure 7: Results of the focal plane coherent light modulation (FPCLM) experiment on a PIAA coronagraph at the Subaru Telescope laboratory. A raw coronagraph image is shown at the upper left. The partially transmitted bright central star is visible at the center of the frame. The D-shaped opening below the bright star is transmitted by the focal plane mask, and is the area where contrast is measured. The average contrast in this region is $2.27 \mathrm{e}-7$ in this frame. The FPCLM successfully separates the light into a coherent component (upper right) and an incoherent component (lower left): as expected, interference fringes due to internal reflection within the CCD entrance window are visible in the coherent portion of the image, but not visible in the incoherent portion. A time-averaged image of the complex amplitude in the focal plane shows that static and slow speckles have been reduced to $3.5 \mathrm{e}-9$ contrast.

\section{CONCLUSION, DISCUSSION OF PERFORMANCE LIMITS}

The wavefront control architecture for the SCExAO system offers a comprehensive solution to well known sources of errors in the current generation of AO systems. Thanks to a dedicated fast pointing control loop, the effect of low order aberrations on coronagraph performance will be mitigated. SCExAO will use a visible WFS optimized for high contrast coronagraphic imaging. With coherent light modulation in the focal plane, slow and static speckles will be removed. SCExAO also delivers a real-time estimate of the scattered light in the focal plane, which can then be subtracted from the science image. Key subsystems for SCExAO have been successfully tested in a laboratory environment: pointing has been controlled to $1 \mathrm{e}-3 \lambda / \mathrm{D}$ and static and slow speckles have been removed at the $3.5 \mathrm{e}-9$ level.

With its nlCWFS, SCExAO should be able to deliver 1e-4 to 1e-5 raw contrast at a few $\lambda / \mathrm{D}$ separation on bright targets. With a simple assumption that the incoming wavefront consists of a purely static (or very slow) term plus a fast atmospheric term, the residual WF aberrations in SCExAO should be free of slow/static speckles, and should consist of 1e-4 to $1 \mathrm{e}-5$ speckles with a coherence time of a few ms. Assuming a 1e-4 to $1 \mathrm{e}-5$ raw contrast with $10 \mathrm{~ms}$ coherence time, the 1-hr contrast limit should then range from $2 \mathrm{e}-7$ to $2 \mathrm{e}-8$, which could potentially be augmented with spectral or polarimetric differential imaging. 
We however note that reaching this level of contrast will also require a very good understanding of the seemingly incoherent light due to chromatic effect and dynamical effects (vibrations for example). The ultimate contrast limit is likely to be a function of how well such effects are understood and removed from the data.

\section{REFERENCES}

[1] Martinache, F., Guyon, O., Vogt, F., Garrel, V., Yokochi, K., Yoshikawa, T., "The Subaru coronagraphic extreme AO (SCExAO) system: progress report", SPIE, 7736-216 (2010)

[2] Garrel, V., Guyon, O., Baudoz, P., Martinache, F., Vogt, F., Yoshikawa, T., Yokochi, K., "The Subaru coronagraphic extreme AO (SCExAO) system: visible imaging mode", SPIE, 7736-222 (2010)

[3] Guyon, O. "Phase-induced amplitude apodization of telescope pupils for extrasolar terrestrial planet imaging", A\&A 404, 379 (2003)

[4] Guyon, O., Pluzhnik, E. A., Kuchner, M. J., Collins, B., Ridgway, S. T. "Theoretical Limits on Extrasolar Terrestrial Planet Detection with Coronagraphs", ApJS 167, 81 (2006)

[5] Guyon, O., Pluzhnik, E.A., Martinache, F., Totems, J., Tanaka, S., Matsuo, T., Blain, C., Belikov, R. "High Contrast Imaging and Wavefront Control with a PIAA Coronagraph: Laboratory System Validation", PASP 122, 71 (2010)

[6] Minowa, Y., Hayano, Y., Oya, S., Watanabe, M., Hattori, M., Guyon, O., Egner, S., Saito, Y., Ito, M., Iye, M., Takami, H., Garrel, V., Colley, S., Golota, T., "Performance of Subaru adaptive optics system AO188", SPIE, 7736- 134 (2010)

[7] Guyon, O. "Limits of Adaptive Optics for High Contrast Imaging", ApJ 629, 592-614 (2005)

[8] Guyon, O. "High Sensitivity Wavefront Sensing with a non-linear Curvature Wavefront Sensor", PASP 122, 49 (2010)

[9] Mateen, M., Garrel, V., Hart, M., Guyon, O. "Results from the laboratory demonstration of the nonlinear curvature wavefront sensor", SPIE, 7736-201 (2010)

[10] Guyon, O., Matsuo, T., Angel, R., "Coronagraphic Low Order Wavefront Sensor: Principle and Application to a Phase-Induced Amplitude Coronagraph" ApJ 693, 75 (2009)

[11] Vogt, F., Guyon, O., Martinache, F., Garrel, V., Yokochi, K., Yoshikawa, T., Matsuo, T. "The Subaru coronographic extreme adaptive optic (SCExAO) system: implementation and performance of the coronographic loworder wave-front sensor", SPIE, 7736-37 (2010)

[12] Give'on, A., Kern, B., Shaklan, S., Moody, D.C., Pueyo, L. "Broadband wavefront correction algorithm for highcontrast imaging systems", SPIE, 6691 (2007) 